Esta experiencia pone la atención en una tecnología de alto costo de uso frecuente en países de ingresos altos y de escasa disponibilidad en economías como la de Argentina. El videolaringoscopio artesanal aparece como una alternativa a ese problema: su costo es más bajo (1900 pesos argentinos) y se puede reutilizar.

La cámara endoscópica se puede ensamblar a ramas de laringoscopios rectos o curvos. Su higiene es similar a la de cualquier laringoscopio convencional.

Se describe aquí una simulación que permitió a los pediatras familiarizarse con una tecnología nueva, que podría tener algún papel en la protección del equipo de salud y en la seguridad de los pacientes.

No se puede trasladar esta experiencia a una recomendación sobre el uso del dispositivo dado que aún no se ha probado su eficacia en el ámbito clínico pediátrico.

Se requiere investigación que aporte evidencia que responda a estos interrogantes.

\section{REFERENCIAS}

1. The Lancet. COVID-19: Protecting health-care workers. Lancet. 2020;395(10228):922.

2. Tran K, Cimon K, Severn M, Pessoa-Silva CL, et al. Aerosol generating procedures and risk of transmission of acute respiratory infections to healthcare workers: a systematic review. PLoS One. 2012;7(4):e35797.

3. Zuo MZ, Huang YG, Ma WH, Xue ZG, et al. Expert Recommendations for Tracheal Intubation in Critically ill Patients with Noval Coronavirus Disease 2019. Chin Med Sci J. 2020;35(2):105-9.
4. Orser BA. Recommendations for Endotracheal Intubation of COVID-19 Patients. Anesth Analg. 2020;130(5):1109-10.

5. Cook TM, El-Boghdadly K, McGuire B, McNarry AF, et al. Consensus guidelines for managing the airway in patients with COVID-19: Guidelines from the Difficult Airway Society, the Association of Anaesthetists the Intensive Care Society, the Faculty of Intensive Care Medicine and the Royal College of Anaesthetists. Anaesthesia. 2020;75(6):785-99.

6. Matava CT, Kovatsis PG, Lee JK, Castro P, et al. Pediatric Airway Management in COVID-19 Patients: Consensus Guidelines from the Society for Pediatric Anesthesia's Pediatric Difficult Intubation Collaborative and the Canadian Pediatric Anesthesia Society. Anesth Analg. 2020;131(1):61-73.

7. Balaban O, Tobias JD. Videolaryngoscopy in Neonates, Infants, and Children. Pediatr Crit Care Med. 2017;18(5): 477-85.

8. Grunwell JR, Kamat PP, Miksa M, Krishna A, et al. Trend and Outcomes of Video Laryngoscope Use Across PICUs. Pediatr Crit Care Med. 2017;18(8):741-9.

9. Grünberg G. Intubación nasotraqueal con "videolaringoscopio artesanal" en paciente con vía aérea dificultosa prevista. Anest Analg Reanim. 2012;25(2):55-60.

10. De Freitas J, Moreno J, Silva M. Prototipo de videolaringoscopio: Wi-Mac-Multivision. Rev Chil Anest 2020;49(2):262-70.

11. Karippacheril JG, Umesh G, Ramkumar V. Inexpensive video-laryngoscopy guided intubation using a personal computer: Initial experience of a novel technique. J Clin Monit Comput. 2014;28(3):261-4.

12. Simulación de Videolaringoscopía en lactante con COVID 19. [Consulta: 26 de mayo de 2020]. Disponible en: https: / / www.youtube.com/watch?v=7eAC6R17GuQ

13. Saito T, Taguchi A, Asai T. Videolaryngoscopy for tracheal intubation in patients with COVID-19. Br J Anaesth. 2020;125(3):e284-6.

14. Hall D, Steel A, Heij R, Eley A, et al. Videolaryngoscopy increases 'mouth-to-mouth' distance compared with direct laryngoscopy. Anaesthesia. 2020;75(6):822-3.

15. Begley JL, Lavery KE, Nickson CP, Brewster DJ. The aerosol box for intubation in coronavirus disease 2019 patients: an in-situ simulation crossover study. Anaesthesia. 2020;75(8):1014-21.

\title{
¿Es el electrocardiograma correctamente interpretado por médicos residentes de Pediatría? Experiencia previa y posterior a una intervención educacional en un hospital universitario
}

\author{
Are electrocardiograms correctly interpreted by pediatric residents? Experience \\ before and after an educational intervention in a teaching hospital
}

\author{
Dra. Georgina Bergero ${ }^{a}$ Dra. Melina J. Saavedra ${ }^{b}$ Dra. Marina Guglielmino ${ }^{a}$, Dra. Amanda R. Soto Pérez ${ }^{a}$, \\ Dr. Julián Llera ${ }^{a}$ y Dr. Julio Busaniche ${ }^{a}$
}

a. Servicio de Clínica Pediátrica, Departamento de Pediatría. b. ServiciodeCardiología Pediátrica, DepartamentodePediatría. Hospital Italiano de Buenos Aires, Ciudad Autónoma de Buenos Aires. Argentina.

Correspondencia:

Dra. Georgina Bergero: bergerogeorgina@gmail.com
Financiamiento: Ninguno.

Conflicto de intereses: Ninguno que declarar.

Recibido: 5-10-2020

Aceptado: 17-12-2020 


\section{RESUMEN}

La incidencia del paro cardíaco pediátrico es desconocida; sus principales etiologías son cardiopatías congénitas, miocardiopatías y arritmias ventriculares. Elelectrocardiograma es un método diagnóstico que podría detectarlas precozmente y disminuir la morbimortalidad.

El objetivo del estudio fue describir las habilidades de residentes dePediatría para reconocer si un electrocardiograma era normal o anormal y realizar un diagnóstico electrocardiográfico preciso, antes y después de una intervención educativa. Participaron médicos residentes de primer año de Pediatría. Se tomó una evaluación con 12 trazados de electrocardiogramas, antes y después de un módulo educativo, y se compararon los puntajes mediante la prueba de " $t$ " para datos pareados.

No se halló diferencia entre ambas evaluaciones para la interpretación de electrocardiogramas como normales o anormales (p: 0,42). Sin embargo, hubo una diferencia estadísticamente significativa en cuanto a los diagnósticos de certeza $(p<0,002)$. Los diagnósticos de certeza electrocardiográficos mejoraron luego de implementar un módulo educacional.

Palabras clave: educación médica, aprendizaje, cardiología, electrofisiología cardíaca.

http:/ / dx.doi.org/10.5546/ aap.2021.273

Texto completo en inglés:

http:/ / dx.doi.org/10.5546/ aap.2021.eng.273

Cómo citar: Bergero G, Saavedra MJ, Guglielmino M, Soto Pérez AR, et al. ¿Es el electrocardiograma correctamente interpretado por médicos residentes de Pediatría? Experiencia previa y posterior a una intervención educacional en un hospital universitario. Arch Argent Pediatr 2021;119(4):273-276.

\section{INTRODUCCIÓN}

El electrocardiograma es uno de los estudios diagnósticos más utilizados, ya que, a través del análisis de la actividad eléctrica cardíaca, es posible detectar múltiples patologías, que incluyen algunas que pueden poner en riesgo la vida. ${ }^{1}$ El paro cardíaco en pediatría es causante de más de 2000 muertes por año en Estados Unidos. ${ }^{2}$ Se desconoce su incidencia a nivel mundial. Las principales causas, a diferencia de la población adulta, son el resultado final de la insuficiencia respiratoria progresiva o el shock. Las etiologías más frecuentes de paro cardíaco de origen primario son cardiopatías congénitas, miocardiopatías y arritmias ventriculares. ${ }^{3}$ En algunos casos, el hallazgo precoz de alteraciones en el electrocardiograma podría permitir establecer un diagnóstico y tratamiento oportuno y, así, disminuir su morbimortalidad.

Estudios recientes describen que la interpretación del electrocardiograma es limitada tanto en médicos como en estudiantes de Medicina. ${ }^{4-9}$ Además, múltiples programas de residencias no cuentan con entrenamiento formal en dicha habilidad. ${ }^{5}$
Los pediatras son, frecuentemente, los primeros agentes de salud en evaluar a un niño con posible patología cardíaca. Es de vital importancia que el profesional cuente con la capacidad de interpretar electrocardiogramas. ${ }^{4}$

En la Argentina, no existen publicaciones que aborden esta problemática. El objetivo del estudio fue describir las habilidades de residentes de Pediatría para reconocer si un electrocardiograma era normal o anormal y para realizar un diagnóstico preciso, antes y después de una intervención educativa.

\section{POBLACIÓN Y MÉTODOS}

Estudio cuasiexperimental de tipo antes y después. Desarrollado entre agosto y septiembre de 2019 en el Departamento de Pediatría en un hospital general universitario donde se realizan, aproximadamente, 128000 consultas pediátricas anuales.

La participación del estudio fue de carácter voluntario. Se invitó a participar a médicos residentes de primer año de Pediatría. Se realizó el proceso de consentimiento informado oral a los que aceptaron participar.

Se tomaron dos exámenes, denominados pretest y postest. El módulo educacional se dictó 30 días antes del postest. Los exámenes contenían 12 trazados de electrocardiogramas seleccionados por una médica cardióloga infantil, los cuales eran representativos de la práctica pediátrica por su frecuencia de aparición o por su gravedad. Incluían 4 trazados normales o variantes de la normalidad y 8 anormales (Tabla 1). Los electrocardiogramas fueron adquiridos en nuestra Institución y constaban de 12 derivaciones (véase el Anexo en formato electrónico).

El nombre y la edad de los pacientes fueron suprimidos. Los exámenes fueron presenciales, con una duración de 60 minutos. Se evaluó la capacidad de discriminar entre resultado normal, variante de la normalidad o anormal a través de la selección de estas opciones, y se colocó un sitio de texto libre para detallar el diagnóstico en caso de identificarlo.

El módulo educacional fue dictado por una médica cardióloga infantil en forma presencial con presentación de diapositivas y tiempo destinado a preguntas. Su duración fue de 70 minutos e incluyó la colocación de electrodos, la confección del sistema hexaxial de Bailey y el análisis de ritmo cardíaco, frecuencia cardíaca, conducción auriculoventricular, características de la ondas, segmentos e intervalos, determinación del eje 
eléctrico y QTc. Se analizaron electrocardiogramas normales y patológicos, que incluían los diagnósticos contemplados en el examen, que utilizaba diferentes trazados.

Se excluyeron los residentes que no completaron ambos test y los que no asistieron al módulo educacional. Los resultados correctos no fueron revelados durante el estudio. El protocolo fue aprobado por el Comité de Ética de nuestra Institución.

\section{Análisis estadístico}

Se determinaron dos puntajes: el primero contempló si el electrocardiograma fue reconocido como normal o anormal, y el segundo, si el diagnóstico escrito en el texto libre fue correcto o incorrecto. Se definió como diagnóstico incorrecto cuando el texto libre fue dejado en blanco o incompleto. Ambos puntajes se expresaron en porcentaje de respuestas correctas por participante, y el mayor porcentaje correspondió a la mayor cantidad de aciertos.

Las variables categóricas se expresaron en número absoluto y frecuencia, y las variables continuas, en media y desvío estándar (DE) con intervalo de confianza del $95 \%$ o mediana y rango intercuartílico según la distribución observada. Se compararon los puntajes mediante la prueba de " $t$ " para datos pareados. Se consideró significancia estadística un valor de $p<0,05$. Se utilizó el software STATA 13.

\section{RESULTADOS}

Durante el período de estudio, 14 médicos residentes de primer año formaban parte de la residencia de Pediatría y aceptaron participar. Sin embargo, 2 fueron excluidos por no concurrir al módulo educacional o no haber realizado el postest. Doce residentes (el $86 \%$ ) fueron enrolados.

Se analizó la interpretación correcta de trazados electrocardiográficos como normales o variantes de la normalidad y anormales, sin hallarse diferencia significativa $(p=0,42)$ entre la media pretest del $78,4 \%$ (DE: 13) y postest del $81,3 \%$ (DE: 6,2). Sin embargo, nuestro estudio mostró una mejoría estadísticamente significativa en cuanto al reconocimiento de los diagnósticos de certeza $(\mathrm{p}<0,002)$ entre la media pretest del $22,2 \%$ (DE: 14,8$)$ y postest del 36,1 \% (DE: 21,1) (Tabla 2).

TABLA 1. Electrocardiogramas seleccionados para los exámenes

Electrocardiogramas normales
Electrocardiograma normal
Taquicardia sinusal
Bradicardia sinusal
Arritmia sinusal
Electrocardiogramas anormales
Hipertrofia ventricular izquierda
Intervalo Qtc prolongado
Síndrome de Wolff-Parkinson-White
Taquicardia ventricular
Bloqueo completo de rama derecha
Aleteo auricular
Extrasístoles ventriculares
Bloqueo auriculoventricular de $2 .{ }^{\text {do }}$ grado

TABLA 2. Porcentaje de respuestas correctas de los residentes pre-y postest

\begin{tabular}{|c|c|c|c|c|}
\hline \multirow[b]{2}{*}{ Residente de $1 .^{\text {er }}$ año } & \multicolumn{2}{|c|}{ Normal/anormal } & \multicolumn{2}{|c|}{ Diagnóstico } \\
\hline & Pretest $(\%)$ & Postest $(\%)$ & Pretest $(\%)$ & Postest $(\%)$ \\
\hline 1 & 66,6 & 83,3 & 33,3 & 50 \\
\hline 2 & 91,6 & 91,6 & 16,6 & 33,3 \\
\hline 3 & 91,6 & 91,6 & 33,3 & 25 \\
\hline 4 & 91,6 & 75 & 16,6 & 25 \\
\hline 5 & 58,3 & 75 & 25 & 50 \\
\hline 6 & 75 & 75 & 16,6 & 16,6 \\
\hline 7 & 66,6 & 83,3 & 8,3 & 8,3 \\
\hline 8 & 75 & 75 & 33,3 & 58,3 \\
\hline 9 & 75 & 75 & 16,6 & 16,6 \\
\hline 10 & 66,6 & 83,3 & 25 & 50 \\
\hline 11 & 100 & 83,3 & 50 & 75 \\
\hline 12 & 83,3 & 83,3 & 58,3 & 58,3 \\
\hline Media (DE) & $78,4(13,0)$ & $81,2(6,2)$ & $22,2(14,8)$ & $36,1(21,1)$ \\
\hline Valor de $p$ & \multirow{2}{*}{\multicolumn{2}{|c|}{$\begin{array}{c}0,42 \\
{[-4,7-10,4]}\end{array}$}} & \multirow{2}{*}{\multicolumn{2}{|c|}{$\begin{array}{c}0,002 \\
{[5,9-21,8]}\end{array}$}} \\
\hline Intervalo de confianza del $95 \%$ & & & & \\
\hline
\end{tabular}

DE: desvío estándar. 


\section{DISCUSIÓN}

Nuestro estudio mostró que la implementación de un módulo educacional en un grupo de residentes de primer año de Pediatría destinado al análisis e identificación de electrocardiogramas pediátricos mejoraba significativamente su habilidad para arribar al diagnóstico correcto. En la bibliografía, un reciente metaanálisis concluyó que la habilidad en la interpretación de electrocardiogramas en ausencia de entrenamiento era baja y que mejoraba luego de intervenciones educativas. En las distintas poblaciones, se observó que, luego del entrenamiento, la mejoría obtenida era menor que la esperada. ${ }^{9}$

Conforme con el estudio de Khanna et al., se mostró una diferencia estadísticamente significativa en la interpretación del diagnóstico de certeza con un aumento del $14 \%$ luego de la intervención educacional. Sin embargo, en nuestra cohorte, no se hallaron diferencias en cuanto a la capacidad de reconocer si un electrocardiograma era normal o anormal. Este hallazgo podría deberse a que, en nuestra población, el porcentaje de respuestas correctas en el pretest (del 78,4\%) fue ampliamente superior comparado con el estudio citado (del 35,2\%). ${ }^{2}$

En la publicación de Escudero et al., obtuvieron resultados similares en cuanto a la identificación correcta del electrocardiograma como normal o anormal, con menor precisión para reconocer el diagnóstico correcto. ${ }^{4}$ Nuestra población presentó puntajes menores que los publicados en lo que respecta al reconocimiento del diagnóstico de certeza. ${ }^{6,7}$ Esto podría corresponderse con que el entrenamiento en las universidades se basaba en la interpretación del electrocardiograma adulto.

La mayor dificultad que tuvo nuestra población fue reconocer diagnósticos como intervalo Qt prolongado, síndrome de WolffParkinson-White, bloqueo auriculoventricular y bloqueo completo de rama derecha, en coincidencia con estudios publicados..$^{2,4,6} \mathrm{Sin}$ embargo, la mayor parte de nuestra población logró identificar los electrocardiogramas como anormales, por lo que, en la práctica clínica, hubiese provocado la consulta pertinente con médicos más experimentados.

En la publicación de Crocetti et al., el mejor predictor para la interpretación del electrocardiograma fue una rotación en Cardiología. ${ }^{8}$ En nuestro programa de residencia, actualmente, no existe una rotación formal en Cardiología.

A pesar de que la interpretación de electrocardiogramas es una tarea compleja incluso para especialistas en cardiología, es de suma importancia que los residentes de Pediatría los interpreten correctamente y que reconozcan entidades que requieran una conducta médica o la derivación al especialista. A partir de este estudio, se considera relevante la implementación anual de un módulo educacional de electrocardiograma en nuestro programa de residencia y el seguimiento durante los años subsiguientes con el objetivo de evaluar la progresión en la adquisición de estas habilidades.

\section{Limitaciones}

Se realizó en un centro y el tamaño de la muestra fue pequeño. Incluyó a médicos de primer año residentes de Pediatría, lo que podría resultar en un sesgo de selección.

En cuanto a la metodología, con el objetivo de cuidar la privacidad de los pacientes, se eliminó su edad de los electrocardiogramas, lo que resultó en una dificultad para su correcta interpretación. Además, se debió aclarar que, en caso de encontrar más de una anomalía, se consignara la más significativa, ya que, por ejemplo, en el electrocardiograma con bloqueo auriculoventricular de segundo grado, algunos residentes respondieron bloqueo incompleto de rama derecha, lo cual no correspondía a la alteración principal. Finalmente, el postest se realizó 30 días después de la intervención, por lo que no se evaluó la capacidad de retener la información a largo plazo.

\section{CONCLUSIÓN}

Nuestra cohorte de residentes presentó buen desempeño al reconocer si un electrocardiograma era normal o anormal. El arribo al diagnóstico de certeza mejoró luego de un módulo educacional. Sin embargo, es necesario implementar más instancias de entrenamiento para alcanzar mejores niveles de precisión diagnóstica.

\section{REFERENCIAS}

1. Rolskov Bojsen S, Räder SBEW, Holst AG, Kayser L, et al. The acquisition and retention of ECG interpretation skills after a standardized web-based ECG tutorial-a randomised study. BMC Med Educ. 2015;15:36.

2. Khanna S, Iyer VR, Vetter VL. Can Pediatric Practitioners Correctly Interpret Electrocardiograms? J Pediatr. 2019;206:113-8

3. Sherwin ED, Berul CI. Sudden Cardiac Death in Children and Adolescents. Card Electrophysiol Clin. 2017;9(4):569-79.

4. Escudero CA, Sanatani S, Wong KK, Templeton CG. Electrocardiogram interpretation by Canadian general paediatricians: Examining practice, accuracy and confidence. Paediatr Child Health. 2014;19(2):77-83.

5. JheetaJS, Narayan O, Krasemann T. Accuracy ininterpreting the paediatric ECG: UK-wide study and the need for improvement. Arch Dis Child. 2014;99(7):646-8.

6. Snyder CS, Bricker JT, Fenrich AL, Friedman RA, et al. Can pediatric residents interpret electrocardiograms? Pediatr Cardiol. 2005;26(4):396-9.

7. De Jager J, Wallis L, Maritz D. ECG interpretation skills of South African emergency medicine residents. Int J Emerg Med. 2010;3(4):309-14.

8. Crocetti M, Thompson R. Electrocardiogram interpretation skills in pediatric residents. Ann Pediatr Cardiol. 2010;3(1):3-7.

9. Cook DA, Oh SY, Pusic MV. Accuracy of Physicians Electrocardiogram Interpretations: A Systematic Review and Meta-analysis. JAMA Intern Med. 2020;180(11):1-11. 


\section{ANEXO \\ ELECTROCARDIOGRAMAS SELECCIONADOS PARA LOS EXÁMENES}

1) Trazado normal

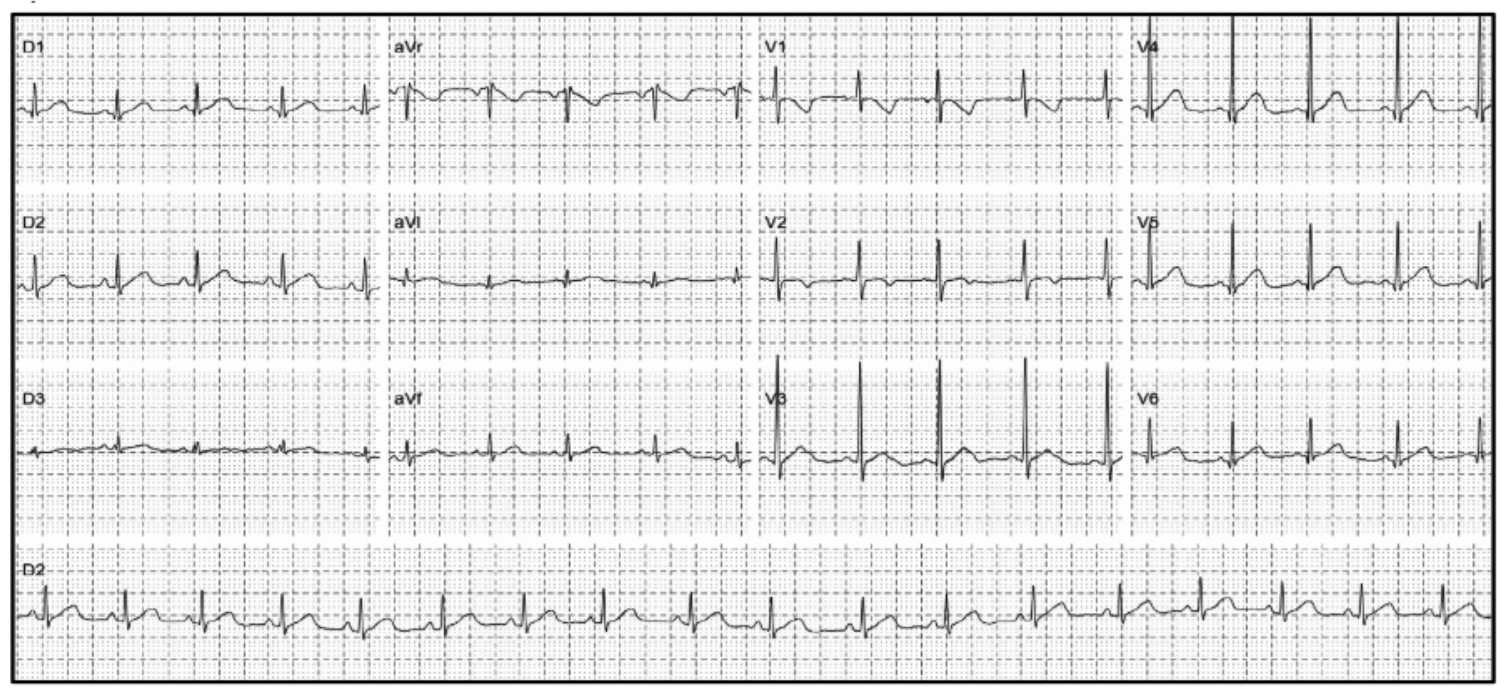

2) Hipertrofia ventricular izquierda

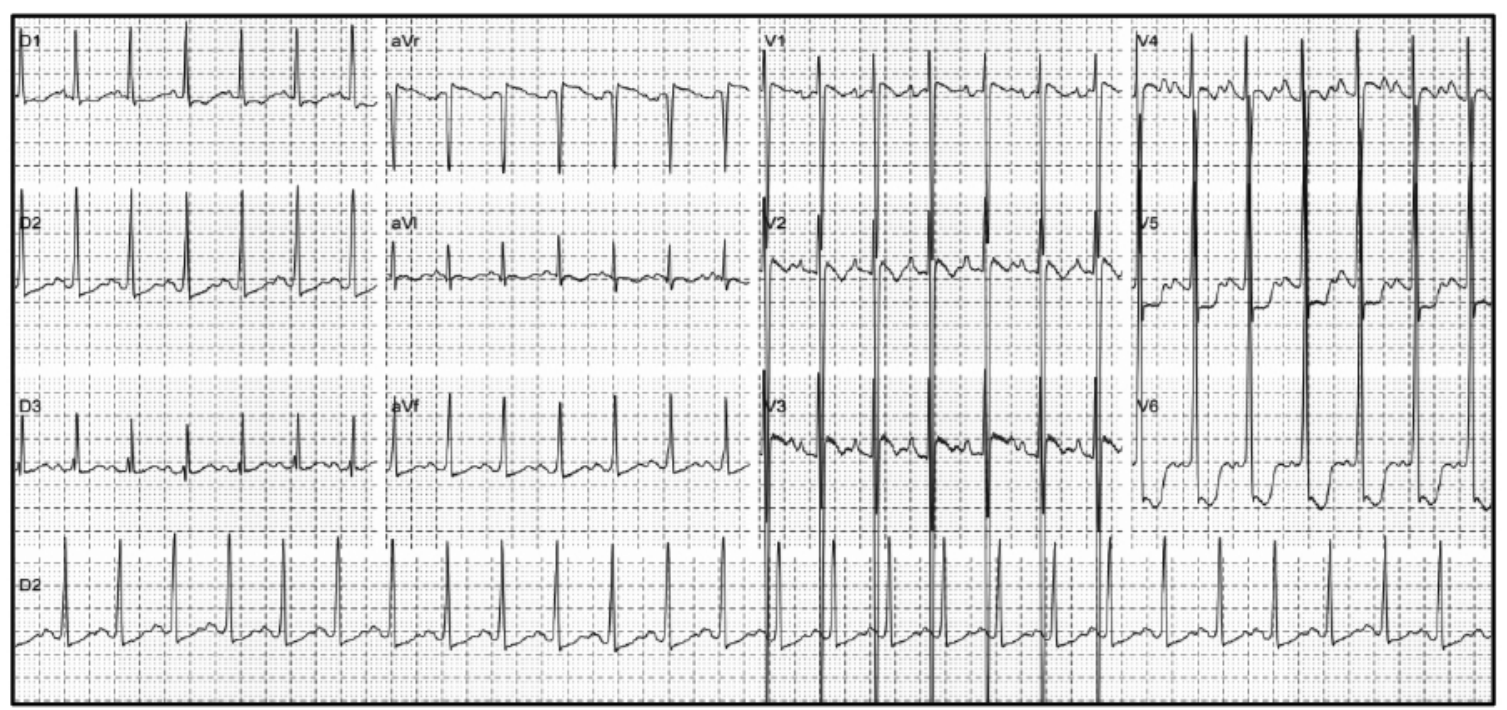




\section{3) Taquicardia sinusal}

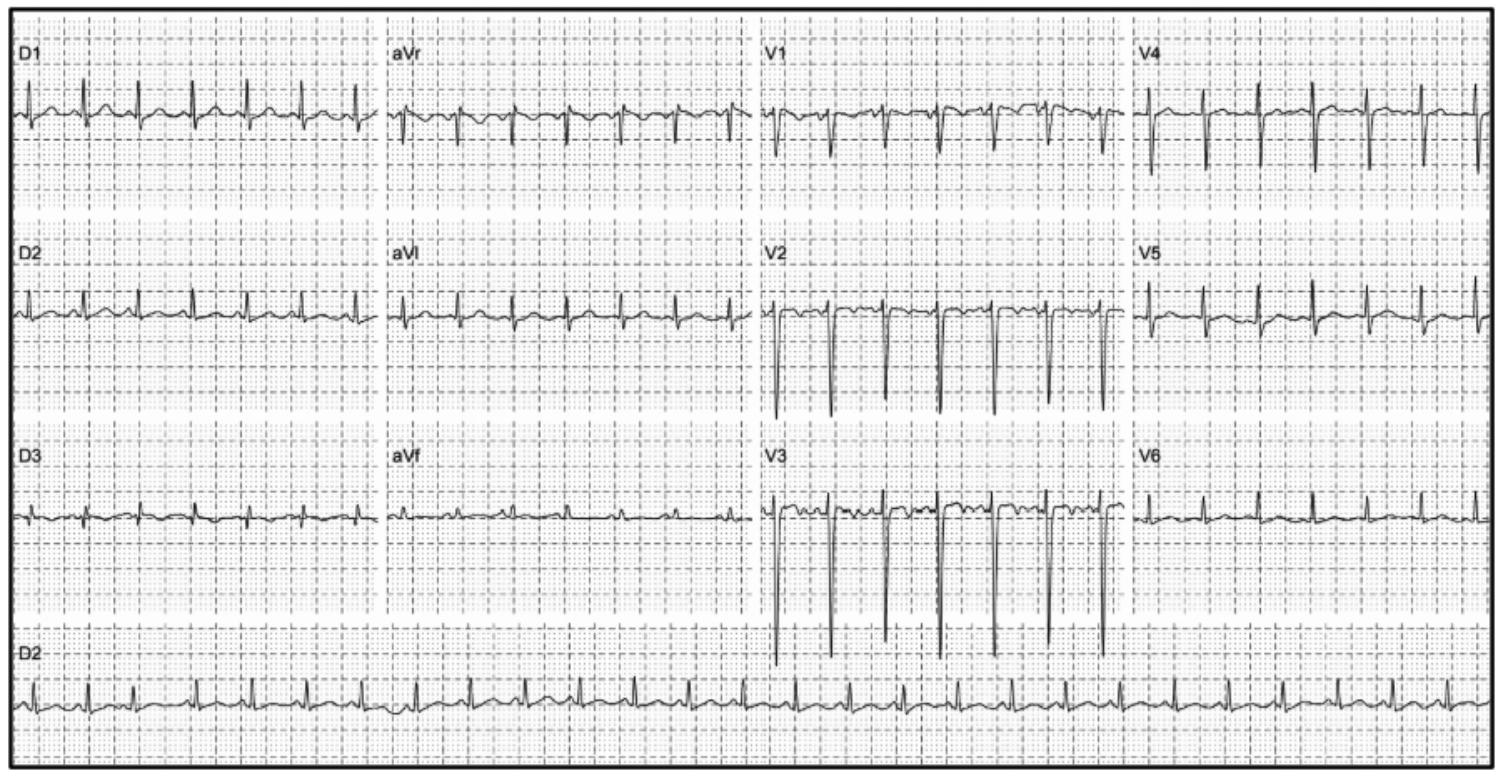

4) Intervalo QTc prolongado

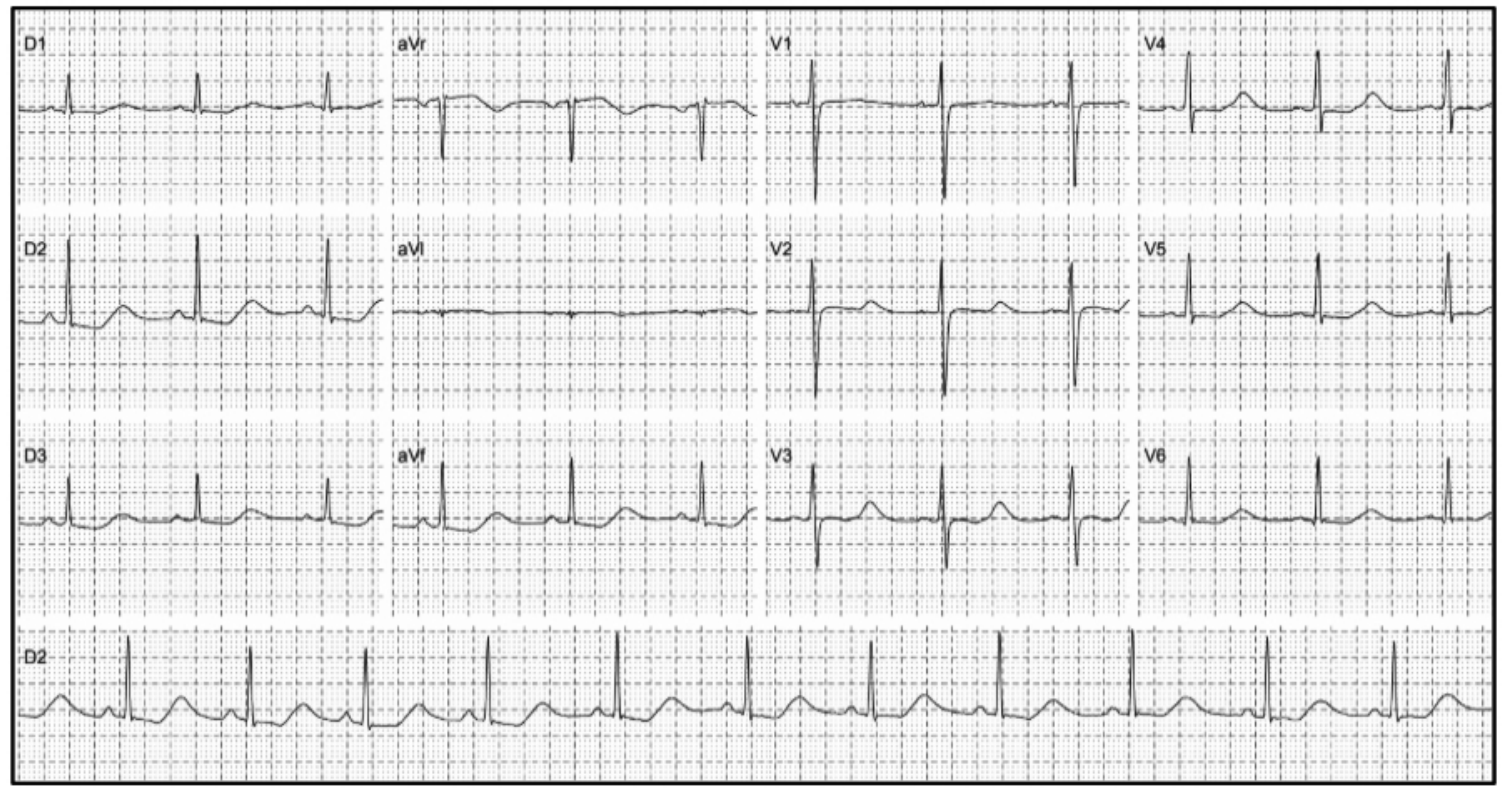


5) Síndrome de Wolff-Parkinson-White

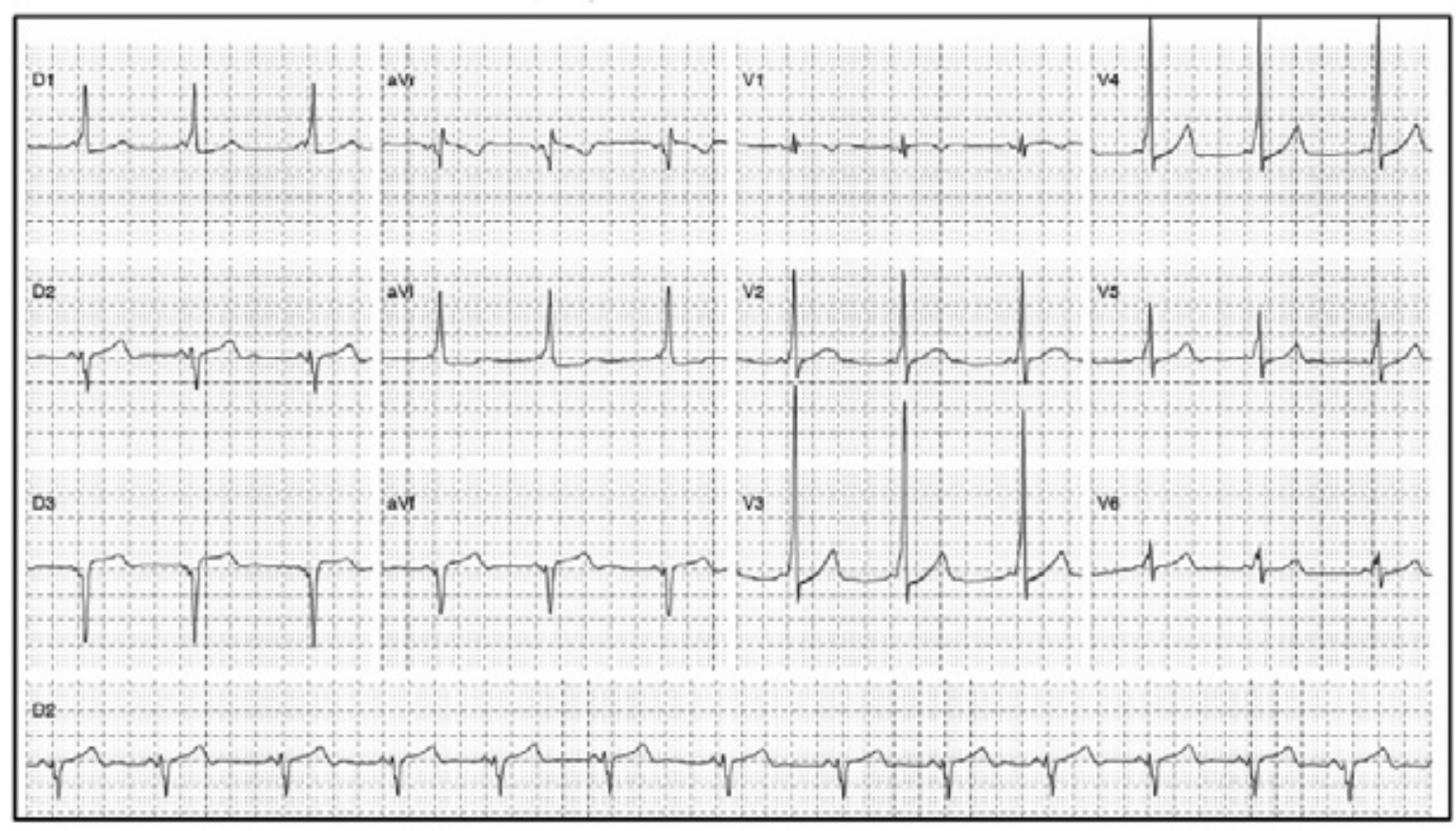

6) Taquicardia ventricular

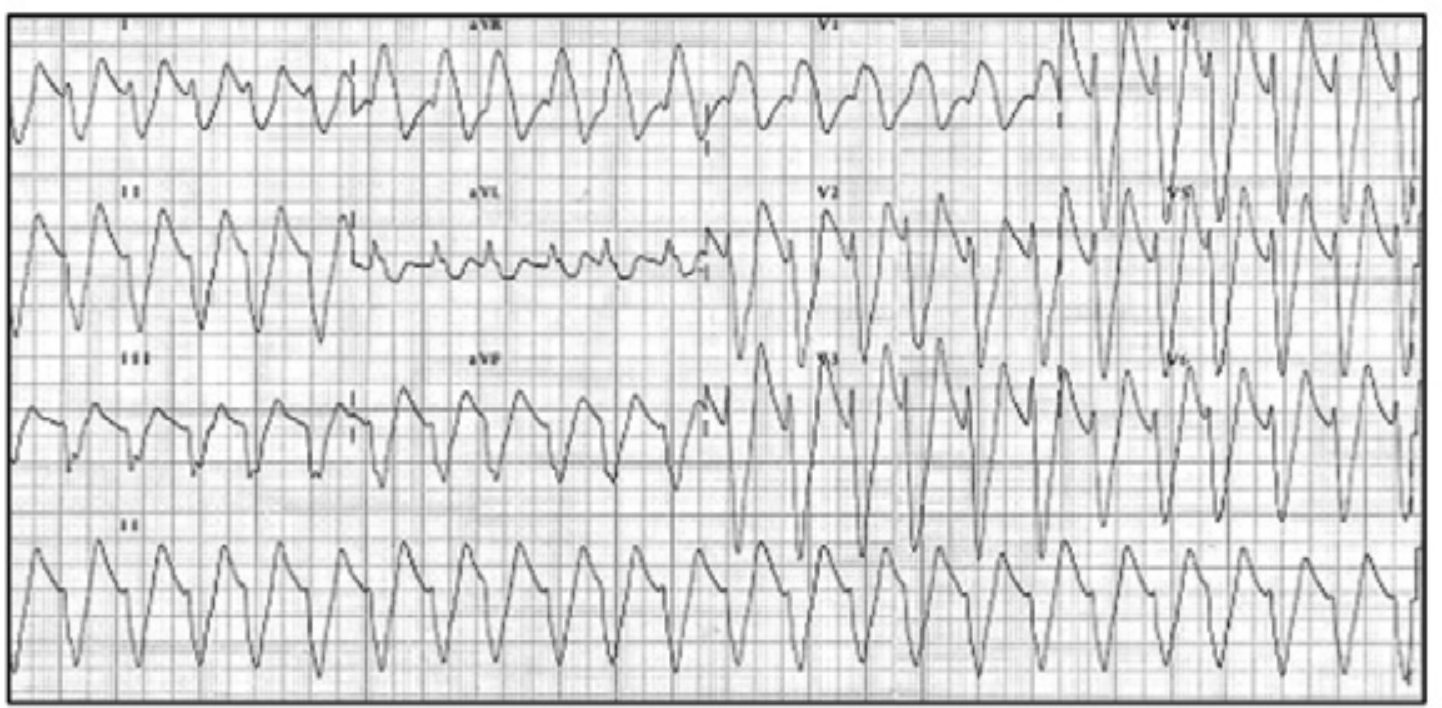


7) Bradicardia sinusal

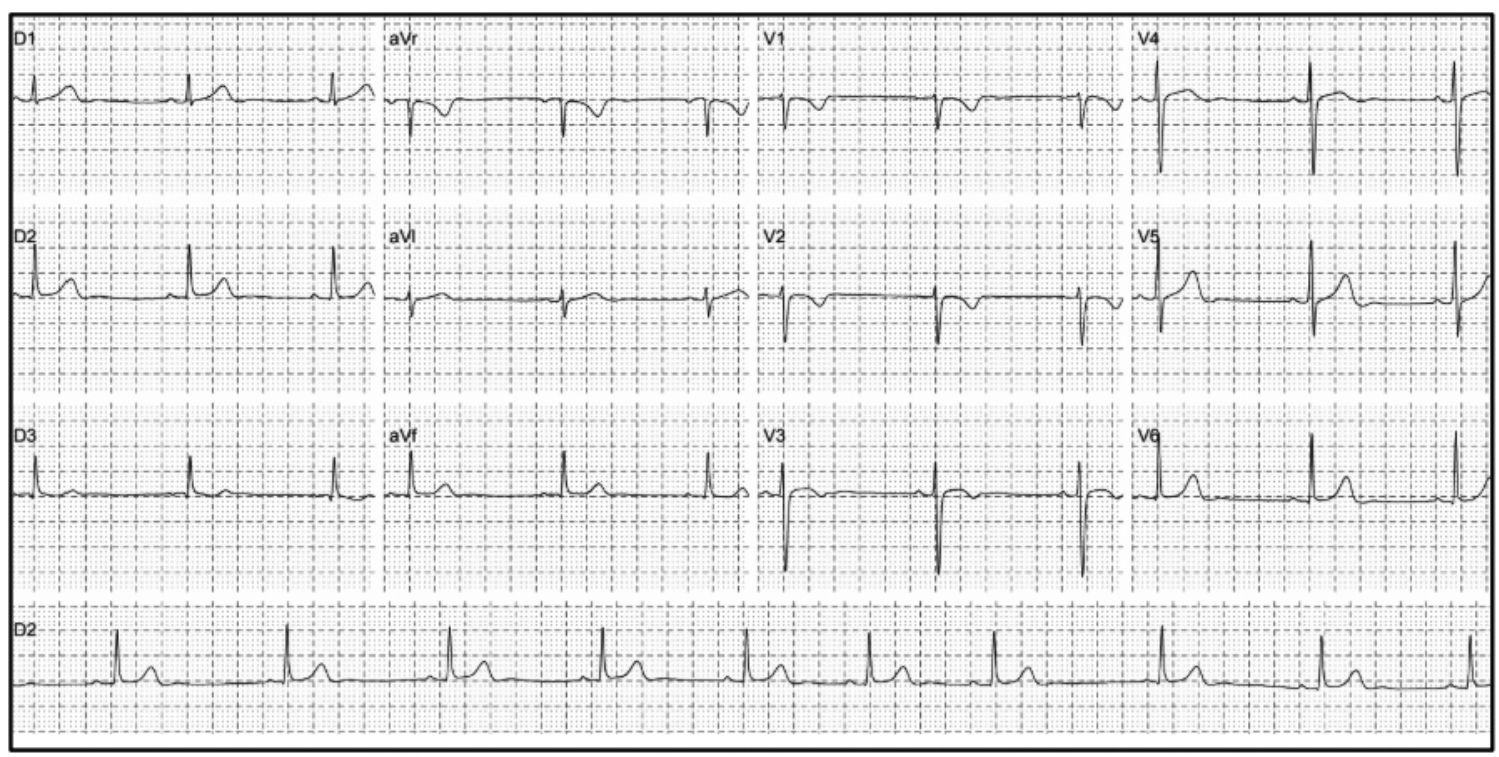

8) Bloqueo completo de rama derecha

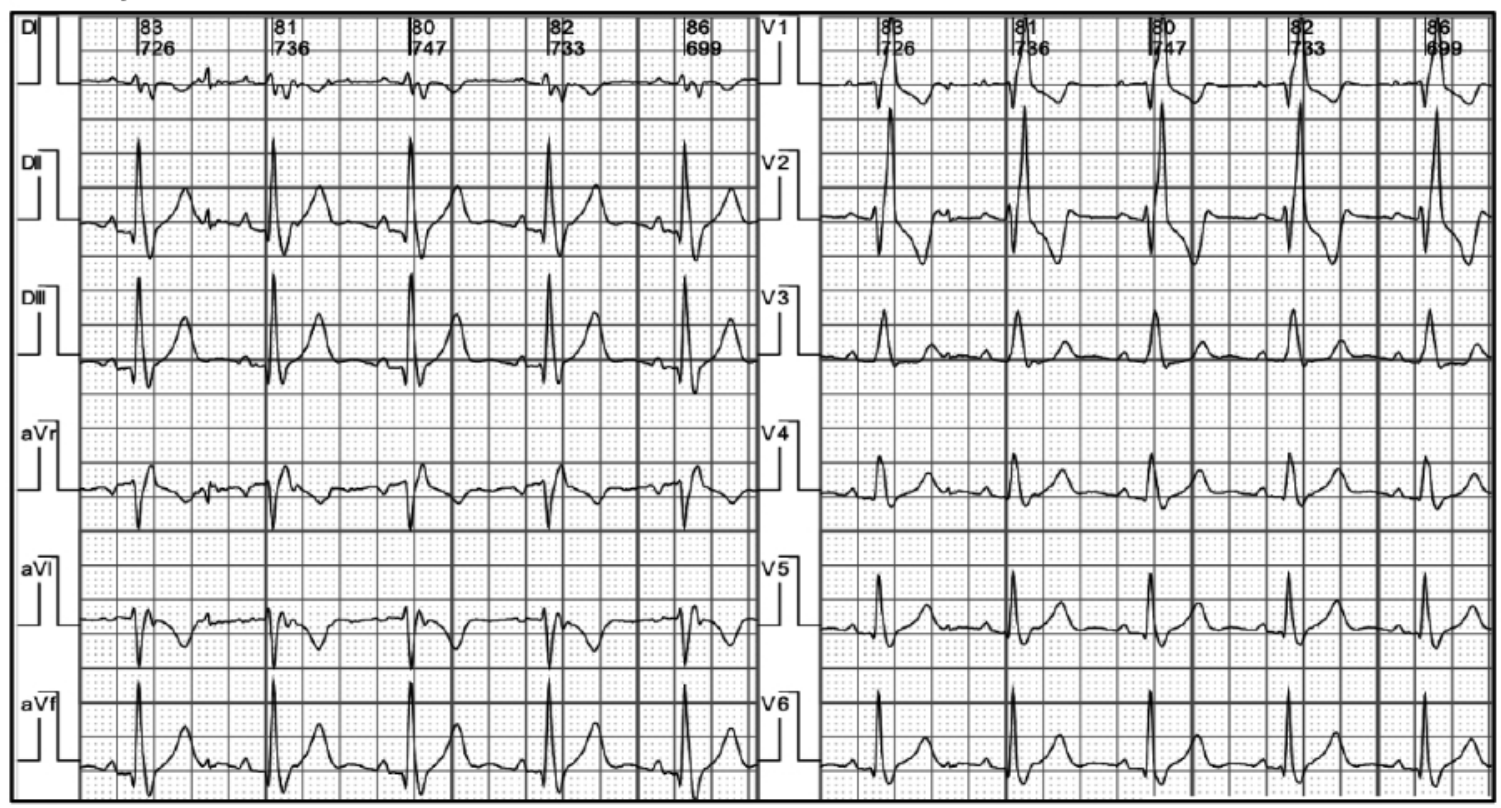




\section{9) Aleteo auricular}

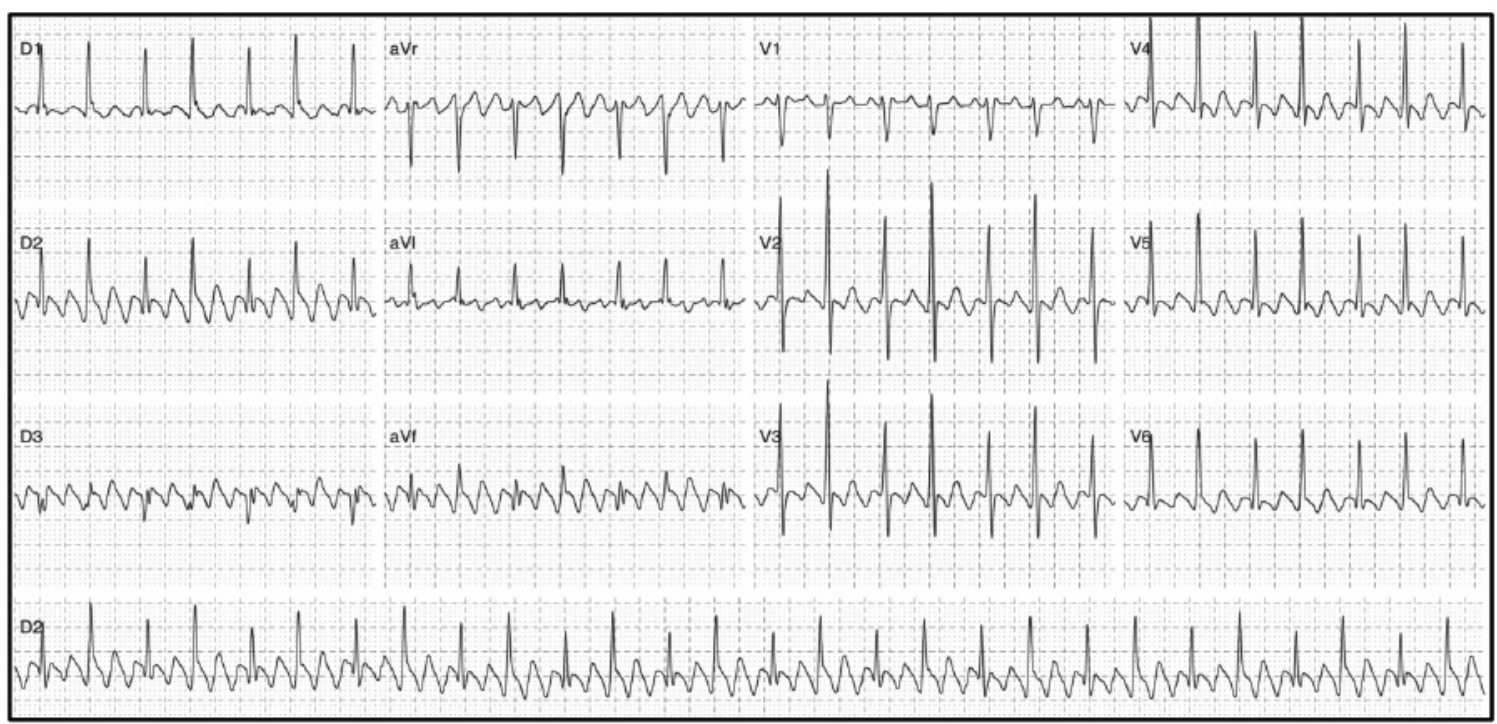

10) Arritmia sinusal respiratoria

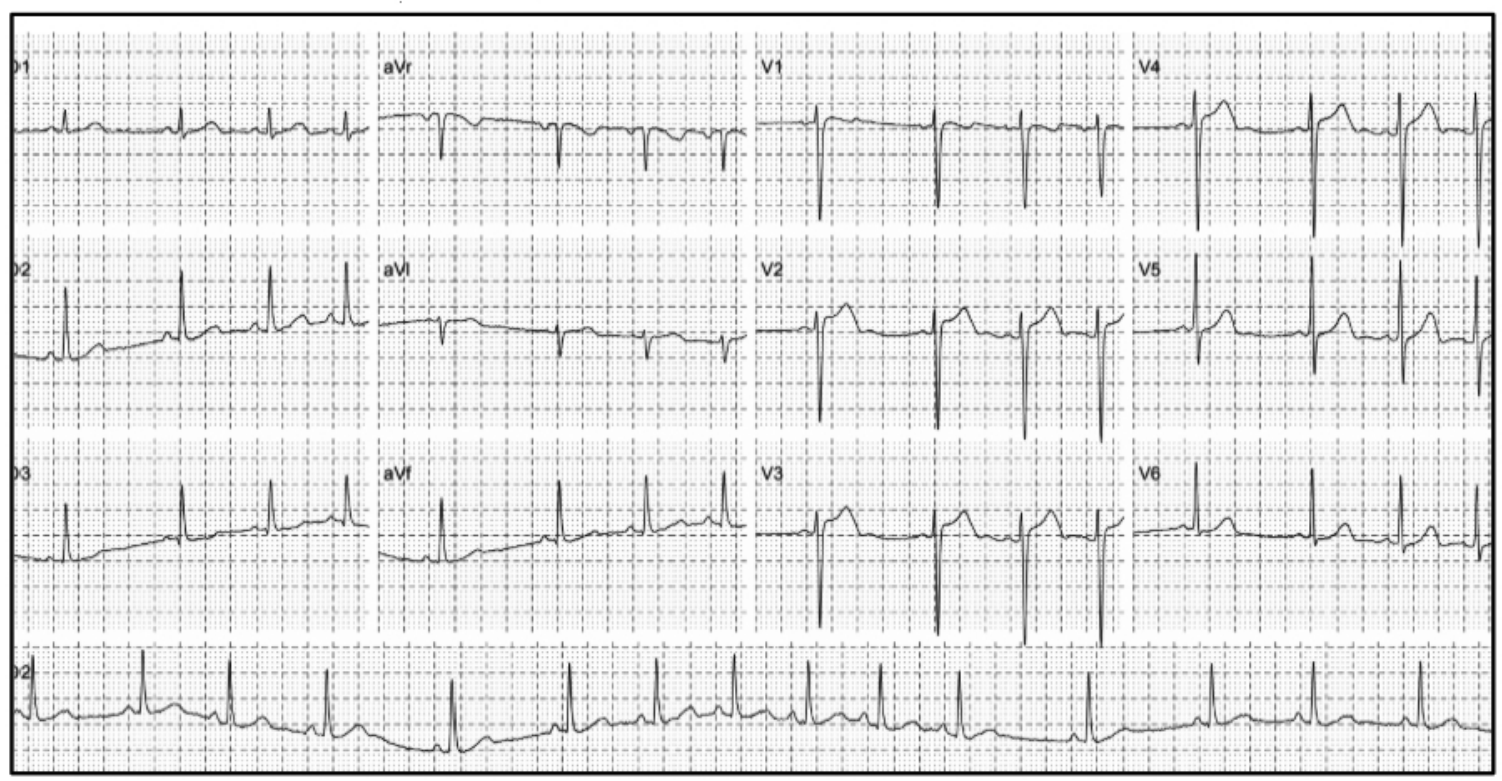


VI / Arch Argent Pediatr 2021;119(4):266-276 / Comunicaciones breves

11) Extrasístoles ventriculares

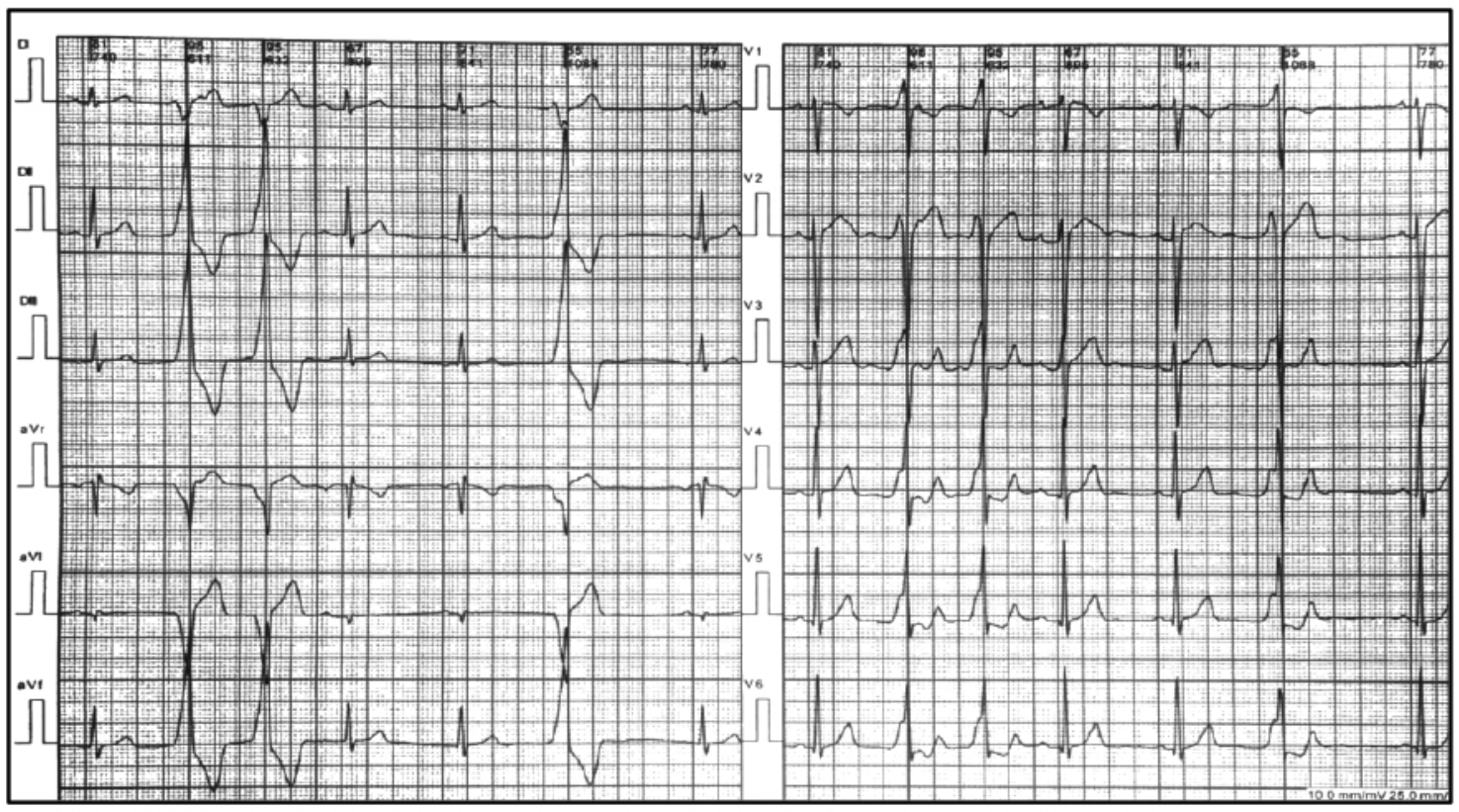

12) Bloqueo auriculoventricular de segundo grado tipo Mobitz 1

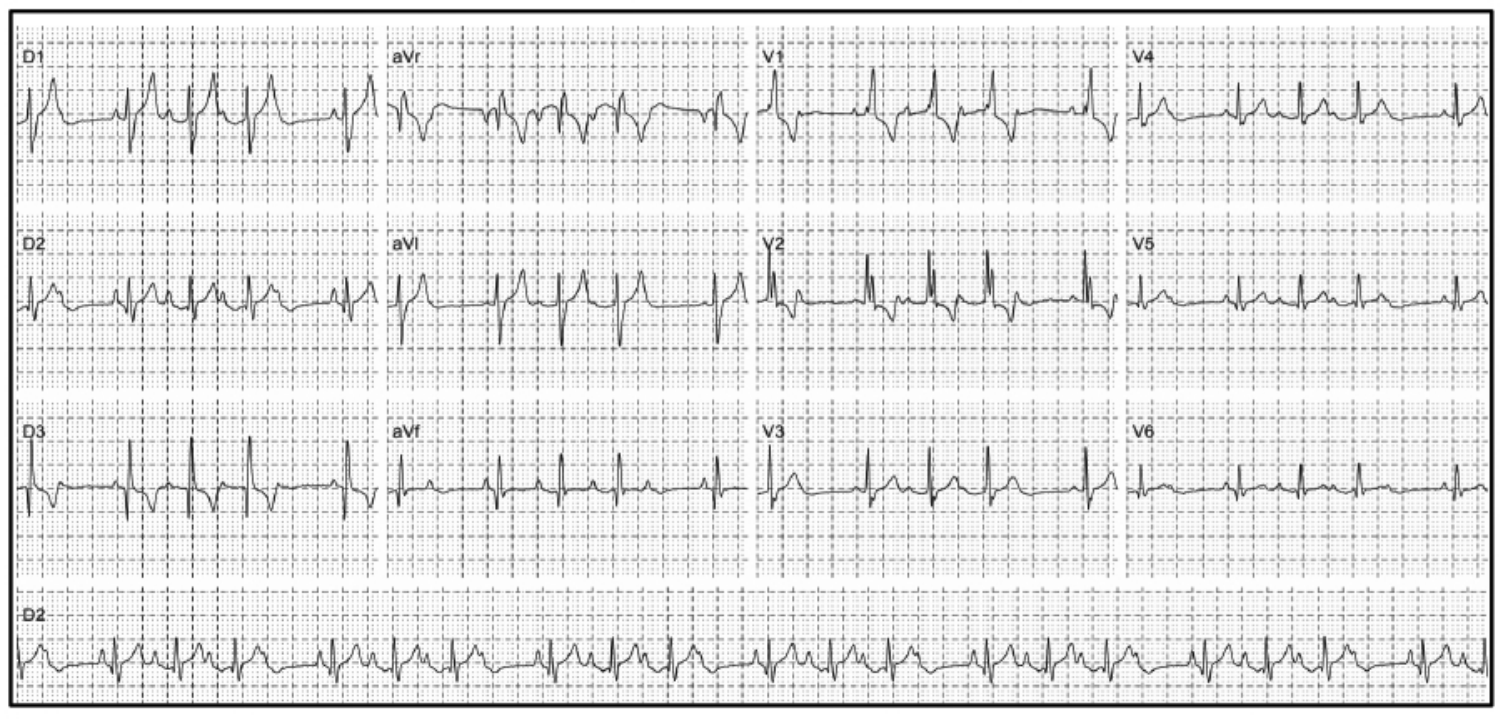

